\title{
Minimal Jumping Emerging Patterns: Computation and Practical Assessment
}

\author{
Bamba Kane, Bertrand Cuissart, and Bruno Crémilleux \\ GREYC - CNRS UMR 6072, \\ University of Caen Basse-Normandie, CS 14032, F-14032 Caen cedex 5 \\ \{bamba.kane, bertrand. cuissart, bruno.cremilleux\}@unicaen.fr
}

\begin{abstract}
Jumping Emerging Patterns (JEP) are patterns that only occur in objects of a single class, a minimal JEP is a JEP where none of its proper subsets is a JEP. In this paper, an efficient method to mine the whole set of the minimal JEPs is detailed and fully proven. Moreover, our method has a larger scope since it is able to compute the essential JEPs and the top-k minimal JEPs. We also extract minimal JEPs where the absence of attributes is stated, and we show that this leads to the discovery of new valuable pieces of information. A performance study is reported to evaluate our approach and the practical efficiency of minimal JEPs in the design of rules to express correlations is shown.
\end{abstract}

Keywords: pattern mining, emerging patterns, minimal jumping emerging patterns, ruled-based classification

\section{Introduction}

Contrast set mining is a well established data mining area [14] which aims at discovering conjunctions of attributes and values that differ meaningfully in their distributions across groups. This area gathers many techniques such as subgroup discovery [17] and emerging patterns [2]. Because of their discriminative power, contrast sets are highly useful in supervised tasks to solve real world problems in many domains $[1,7,12]$.

Let us consider a dataset of objects partitioned into several classes, each object being described by binary attributes. Initially introduced in [2], emerging patterns (EPs) are patterns whose frequency strongly varies between two datasets. A Jumping Emerging Pattern (JEP) is an EP which has the notable property to occur only in a single class. JEPs are greatly valuable to obtain highly accurate rule-based classifiers $[8,9]$. They are used in many domains like chemistry [12], knowledge discovery from a database of images [7], predicting or understanding diseases [3], or DNA sequences [1]. A minimal JEP designates a JEP where none of its proper subsets is a JEP. Minimal JEPs are of great interest because they capture the vital information that cannot be skipped to characterize a class. Using more attributes may not help and even add noise in a classification purpose. Mining minimal JEPs is a challenging task because it is 
a time consuming process. Current methods require either a frequency threshold [4] or a given number of expected patterns [16]. On the contrary, one of the results of this paper is to be able to compute the whole set of minimal JEPs.

The contribution of this paper can be summarized as follows. First, we introduce an efficient method to obtain all minimal JEPs. A key idea of our method is to introduce an alternative definition of a minimal JEP which stems from the differences between pairs of objects, each of a different class. A backtrack algorithm for computing all minimal JEPs is detailed and the related proofs are provided. Our method does not require either a frequency threshold or a number of patterns to extract. It provides a general approach and its scope encompasses the essential JEPs [4] (i.e., JEPs satisfying a given minimal frequency threshold) and the $k$ most supported minimal JEPs [16] which constitute the state of the art in this field. Second, taking into account the absence of attributes may provide interesting pieces of knowledge to build more accurate classifiers as experimentally shown by Terlecki and Walczak [15]. We address this issue. Our method integrates the absence of attributes in the process by adding their negation. It produces the whole set of minimal JEPs both with the present and absent attributes. Practical results advocate in favor of this addition of negated attributes in the description of the objects. Third, the results of an experimental study are given. We analyze the computation of the minimal JEPs, including the absence of attributes and comparisons with essential JEPs and top-k minimal JEPs. Finally, we experimentally assess the quality of minimal JEPs, essential JEPs and top- $k$ minimal JEPs as correlations between a pattern and a class.

Section 2 gives the preliminaries. The description of our method is provided in Section 3. Section 4 presents the experiments. We review related work in Section 5 and we round up with conclusions and perspectives in Section 6.

\section{Preliminaries}

Let $\mathcal{G}$ be a dataset, a multiset consisting of $n$ elements, an element of $\mathcal{G}$ is named an object. The description of an object is given by a set of attributes, an attribute being an atomic proposition which may hold or not for an object. The finite set of all the attributes occurring in $\mathcal{G}$ is denoted by $\mathcal{M}$. In the remainder of this text, for the sake of simplicity, the word "object" is also used to designate the description of an object.

A pattern denominates a set of attributes, an element of the power set $\mathcal{M}$, denoted $\mathcal{P}(\mathcal{M})$. A pattern is included in the object $g$ if $p$ is a subset of the description of $g: p \subseteq g$. The extent of a pattern $p$ in $\mathcal{G}$, denoted $p_{\mathcal{G}}^{\prime}$, corresponds to the set of the objects that include $p: p_{\mathcal{G}}^{\prime}=\{g \in \mathcal{G}: p \subseteq g\}$. A pattern is supported if it is included in at least one object of the dataset. Moreover, we define a relation, $I$, on $\mathcal{G} \times \mathcal{P}(\mathcal{M})$ as follows: for any object $g$ and any pattern $p, g I p \Longleftrightarrow p \subseteq g$.

Usual data mining methods only consider the presence of attributes. With binary descriptions, the absence of an attribute can be explicitly denoted by adding the negation of this attribute in order to build patterns conveying this 
Table 1. A dataset of 6 objects

\begin{tabular}{|c|c|c|c|c|c|}
\hline $\mathrm{Ob}$ & ttri & $1 \neg 1$ & $2 \neg 2$ & $3 \neg 3$ & $4 \neg 4$ \\
\hline $\mathcal{G}_{+}$ & $\begin{array}{l}\mathbf{g}_{1} \\
\mathbf{g}_{2} \\
\end{array}$ & $\begin{array}{ll}x & \\
& x \\
\end{array}$ & $x^{x}$ & $\begin{array}{l}\mathrm{X} \\
\mathrm{x}\end{array}$ & $\mathrm{x}^{\mathrm{x}} \mathrm{x}$ \\
\hline $\mathcal{G}_{-}$ & $\begin{array}{l}\mathbf{g}_{3} \\
\mathbf{g}_{4} \\
\mathbf{g}_{5} \\
\mathbf{g}_{6}\end{array}$ & $\begin{array}{r}\mathrm{X} \\
\mathrm{x} \\
\mathrm{x} \\
\mathrm{x}\end{array}$ & \begin{tabular}{|lll} 
& & \\
$x$ & & \\
& $x$ \\
$x$ & \\
\end{tabular} & \begin{tabular}{|l}
$\mid$ \\
$x$ \\
$x$ \\
$x$ \\
$x$
\end{tabular} & \begin{tabular}{|lll} 
& & \\
$\mathrm{X}$ & \\
$\mathrm{x}$ & \\
& $\mathrm{x}$ \\
\end{tabular} \\
\hline
\end{tabular}

Table 2. Differences from the dataset in Table 1

\begin{tabular}{|c|c|c|c|c|}
\hline & $\mathrm{g}_{3}$ & $\mathrm{~g}_{4}$ & $\mathrm{~g}_{5}$ & $\mathrm{~g}_{6}$ \\
\hline $\mathrm{g}_{1}$ & $1,3, \neg 2$ & $1, \neg 2$ & 1 & $\neg 2,4$ \\
$\mathrm{~g}_{2}$ & $3, \neg 4$ & $\neg 4$ & $2, \neg 4$ & $\neg 1$ \\
\hline $\mathcal{D}_{\bullet}$ & $1,3, \neg 2, \neg 4$ & $1, \neg 2, \neg 4$ & $1,2, \neg 4$ & $\neg 1, \neg 2,4$ \\
\hline
\end{tabular}

information. We integrate this idea in this paper by adding the negation of absent attributes and thus the description of an object always mentions every attribute either positively or negatively. In other words, $\mathcal{M}$ explicitly contains the negation of any of its attributes, the symbol $\neg$ is used to denote the negation of an attribute (cf. Table 1 as an example).

Minimal Jumping Emerging Pattern. We now suppose that the dataset $\mathcal{G}$ is partitioned into two subsets $\mathcal{G}_{+}$and $\mathcal{G}_{-}$, every subset of such a partition is usually named a class of the dataset. We call an object of $\mathcal{G}_{+}$a positive object and an object of $\mathcal{G}_{-}$a negative object. We say that a supported pattern $p$ is a $J E P$ if it is never included in any negative object: $p_{\mathcal{G}}^{\prime} \neq \emptyset$ and $p_{\mathcal{G}}^{\prime} \subseteq \mathcal{G}_{+}$.

A JEP is minimal if it does not contain another JEP as a proper subset. The set of the minimal JEPs is a subset of the set of the JEPs which groups all the most general JEPs. As a JEP contains at least one minimal JEP, when an object includes a JEP then it includes a minimal JEP.

Table 1 displays a dataset of 6 objects partitioned in two datasets: $\mathcal{G}_{+}=$ $\left\{g_{1}, g_{2}\right\}$ and $\mathcal{G}_{-}=\left\{g_{3}, g_{4}, g_{5}, g_{6}\right\}$. The pattern $p=\{1, \neg 2\}$ is a JEP as $p_{\mathcal{G}_{+}}^{\prime}=$ $\left\{g_{1}\right\}$ and $p_{\mathcal{G}_{-}}^{\prime}=\emptyset$ and $\{1\}$ and $\{\neg 2\}$ are not JEPs, $p$ is thus a minimal JEP.

\section{Contribution}

Section 3.1 introduces the key notion of a difference between two objects, it provides a new definition of a minimal JEP. The latter is the support of our algorithm for extracting minimal JEPs which is detailed and proven in Section 3.2.

\subsection{A relation between the minimal JEPs and the differences between objects}

Let $\mathcal{G}$ be a dataset partitioned into two subsets $\mathcal{G}_{+}$and $\mathcal{G}_{-}$. The difference between an object $i$ and an object $j$ groups the attributes of $i$ that are not satisfied by $j: \mathcal{D}_{i, j}=i \backslash j=\{m \in \mathcal{M}: i I m$ and $\neg j I m\}$. When one focuses on a negative object $j$, the gathering of the differences for a negative object $j$ corresponds to the union of the differences between $i$ and $j$, for any positive object $i: \mathcal{D}_{\bullet j}=\cup_{i \in \mathcal{G}_{+}} \mathcal{D}_{i, j}$. In Table 2, the gathering of the differences for the negative object 4 is $\mathcal{D}_{\bullet 4}=\mathcal{D}_{1,4} \cup \mathcal{D}_{2,4}=\{1, \neg 2\} \cup\{\neg 4\}=\{1, \neg 2, \neg 4\}$.

The following lemma is a direct consequence of the definition of the gathering of the differences for a negative object. 
Lemma 1. Let $j$ be a negative object and $p$ be a pattern. If $\mathcal{D}_{\bullet} \cap \cap p \neq \emptyset$ then $p$ is not included in $j: \neg(j I p)$.

It follows that, if a supported pattern $p$ intersects with every gathering of the differences for a negative object and, thanks to Lemma 1, $p$ cannot be included in any negative object, thus $p$ is a JEP. We now reason by contraposition and we suppose that a supported pattern $p$ does not intersect with the gathering of the differences for one negative object $j_{0}: \mathcal{D}_{\bullet} j_{0} \cap p=\emptyset$. If $p$ is supported by a positive object $i_{0}$, as $\mathcal{D}_{\bullet} j_{0} \cap p=\emptyset$ implies $\mathcal{D}_{i_{0}, j_{0}} \cap p=\emptyset$, then $p$ is supported by $j_{0}$. Thus $p$ cannot be a JEP.

A JEP corresponds to a supported pattern which has at least one attribute in every $\mathcal{D}_{\bullet}$, for $j$ a negative object. Proposition 1 follows:

Proposition 1. A supported pattern $p$ is a JEP if $\mathcal{D}_{\bullet} \cap p \neq \emptyset, \forall j \in \mathcal{G}_{-}$

On the example, the JEP $p=\{1, \neg 2\}$ intersects with every $\mathcal{D}_{\bullet}$ (see Table $2): \mathcal{D}_{\bullet} g_{3} \cap p=\{1, \neg 2\}, \mathcal{D}_{\bullet} g_{4} \cap p=\{1, \neg 2\}, \mathcal{D} \bullet g_{5} \cap p=\{1\}$ and $\mathcal{D}_{\bullet} g_{6} \cap p=\{\neg 2\}$.

We now establish a relation between the gathering of the differences and the minimal JEPs.

Proposition 2. A JEP $p$ is a minimal JEP if, for every attribute a of $p, \exists j \in$ $\mathcal{G}_{-}$such that $p \cap \mathcal{D}_{\bullet} j=\{a\}$.

On the example, the JEP $p=\{3,1, \neg 2\}$ is not a minimal JEP since it contains the JEP $\{1, \neg 2\}$. Proposition 2 gives another point of view: since no intersection between $p$ and a $\mathcal{D}_{\bullet j}$ (for $j$ a negative object) corresponds to $\{3\}$, the attribute $\{3\}$ does not play a necessary part in the discriminative power of $p$, thus $p$ is not a minimal JEP.

Proof (of Proposition 2). Let $p$ be a JEP.

Suppose $p$ is not minimal: there exists a JEP $q$, different from $p$, such that $q \subsetneq p$. Consider an attribute $a$ such that $a \in p \backslash q$. As $q$ is a JEP, Prop. 1 imposes that $\forall j \in \mathcal{G}_{-}, q \cap \mathcal{D}_{\bullet j} \neq \emptyset$, it ensues that $\forall j \in \mathcal{G}_{-}, p \cap \mathcal{D}_{\bullet} \neq \neq\{a\}$. One now can state that, if $p$ is not minimal, then $p$ contains one attribute $a$ such that $\forall j \in \mathcal{G}_{-}, p \cap \mathcal{D}_{\bullet} j \neq\{a\}$.

Conversely, suppose there exists an attribute $a$ in $p$ such that $\forall j \in \mathcal{G}_{-}, p \cap$ $\mathcal{D}_{\bullet j} \neq\{a\}$. As $p$ is a JEP, Prop. 1 ensures that $\mathcal{D}_{\bullet} \cap p \neq \emptyset, \forall j \in \mathcal{G}_{-}$. It follows that, $\forall j \in \mathcal{G}_{-}, \mathcal{D}_{\bullet} \cap p \backslash\{a\} \neq \emptyset$. By applying Prop. $1, p \backslash\{a\}$ is a JEP and $p$ cannot be minimal.

Prop. 2 states that a minimal JEP is a supported pattern that excludes all the negative objects and where every attribute is necessary to exclude (at least one) object. It follows:

Consequence of Prop. 2. Let $p$ be a minimal JEP for the dataset $\mathcal{G}_{+} \cup \mathcal{G}_{-}$and $g_{-} \in \mathcal{G}_{-}$. If $p$ is not a minimal JEP for the dataset $\mathcal{G}_{+} \cup \mathcal{G}_{-} \backslash\left\{g_{-}\right\}$then there exists a unique attribute $a, a \in p$, such that $p \backslash\{a\}$ is a minimal JEP for the dataset $\mathcal{G}_{+} \cup \mathcal{G}_{-} \backslash\left\{g_{-}\right\}$. 


\subsection{Calculation of the minimal JEPs}

We now introduce a structure designed to generate all the minimal JEPs for a dataset: a rooted tree whose "valid" leaves are in a one-to-one correspondence with the minimal JEPs. We suppose here that for $\forall j \in \mathcal{G}_{-}, \mathcal{D}_{\bullet j} \neq \emptyset$, as it follows from Prop. 1 that this condition is a necessity for the existence of at least one minimal JEP. We also assume that an arbitrary order is given on the negative objects: for two negative objects $j$ and $j^{\prime}, j \prec j^{\prime}$ if $j$ is accounted before $j^{\prime}$.

Rooted tree. A rooted tree $(T, r)$ is a tree in which one node, the root $r$, is distinguished. In a rooted tree, any node of degree one, unless it is the root, is called a leaf. If $\{u, v\}$ is an edge of a rooted tree such that $u$ lies on the path from the root to $v$, then $v$ is a child of $u$. An ancestor of $u$ is any node of the path from the root to $u$. If $u$ is an ancestor of $v$, then $v$ is a descendant of $u$, and we write $u \leqslant v$; if $u \neq v$, we write $u<v$.

A tree of the minimal JEPs. We create the tree $(T, r)$ as a rooted tree in which each node $x$, except the root $r$, holds two labels: an attribute, $l_{\text {attr }}(x) \in \mathcal{M}$, and a negative object $l_{o b j}(x) \in \mathcal{G}_{-}$. For a node $x$ of $(T, r), B r(x)$ gathers the attributes that occur along the path from the root to $x: B r(x)=\left\{l_{\text {attr }}(y), y \leqslant x\right\}$; $B r(x)$ indicates the pattern considered at $x$. For any node $x$ of $T$ and any attribute $a, a \in B r(x), \operatorname{crit}(a, x)$ gathers the negative objects already considered at the level of $x$ and whose exclusion is due to the sole presence of $a$ in $B r(x)$ : $\operatorname{crit}(a, x)=\left\{j \preceq l_{o b j}(x): \mathcal{D}_{\bullet j} \cap \operatorname{Br}(x)=\{a\}\right\}$.

Definition 1 (A tree of the minimal JEPs (ToMJEPs)). A rooted tree $(T, r)$ is a tree of the minimal JEPs for $\mathcal{G}$ if:

i) any node $x$, except the root $r$, holds two labels: an attribute label, $l_{\text {attr }}(x) \in$ $\mathcal{M}$, and a negative object label, $l_{\text {obj }}(x) \in \mathcal{G}_{-}$.

ii) if $x$ is an internal node then:

a) the children of $x$ hold the same negative object label: $l_{o b j}(y)=\min \{j \in$ $\left.\mathcal{G}_{-}: \mathcal{D}_{\bullet} j \cap \operatorname{Br}(x)=\emptyset\right\}, \forall y$ a child of $x$,

b) every child of $x$ holds a different attribute label,

c) the union of the attribute labels of the children $y$ of $x$ corresponds to $\mathcal{D} \bullet_{o b j}(y)$.

iii) $x$ is a leaf if it satisfies one of the following conditions:

a) $\exists z \preceq x$ such that $\operatorname{crit}\left(l_{a t t r}(z), x\right)=\emptyset$,

b) $\forall j \in \mathcal{G}_{-}, \mathcal{D}_{\bullet} \cap \operatorname{Br}(x) \neq \emptyset$.

A leaf which satisfies the criteria iii)a) is named dead-end leaf, otherwise it is named a candidate leaf.

Figure 1 depicts a ToMJEPs for the dataset of Tables 1 and 2. The nodes with a dashed line are the dead-end leaves, the nodes surrounded by a solid line the candidate leaves. A candidate leaf surrounded by a bold plain line is associated to a supported pattern: it represents a minimal JEP. For example, the node $x$ such that $\operatorname{Br}(x)=\{1, \neg 2\}$ is associated to a minimal JEP while the node 


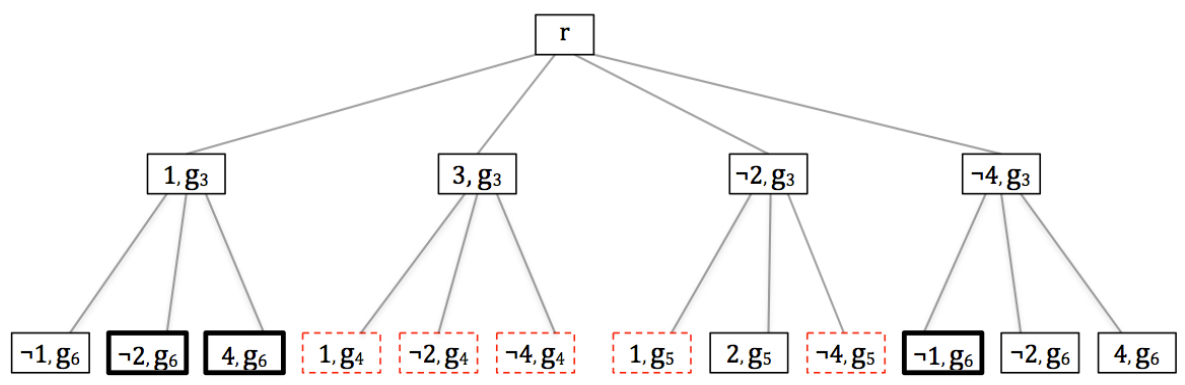

Fig. 1. Example of a tree for minimal JEPs

$y$ such that $\operatorname{Br}(y)=\{\neg 4, \neg 2\}$ is associated to a pattern which is not supported by the dataset. The node $z$ such that $\operatorname{Br}(z)=\{3, \neg 2\}$ is a dead-end leaf: since $\forall j \in\left\{g_{3}, g_{4}\right\},\{3, \neg 2\} \cap \mathcal{D}_{\bullet} \neq \neq\{3\}$, the attribute 3 does not fulfill the constraint raised by Prop. 2, thus crit $(3, z)=\emptyset$.

We will now demonstrate that there is a one-to-one mapping between the "supported" candidate leaves of a ToMJEPs and the minimal JEPs. The following lemma is an immediate consequence of the definition of a ToMJEPs, together with the application of Prop. 1 and 2.

Lemma 2. Let $(T, r)$ be a ToMJEPs and $x$ be a node of $T$, different from a deadend leaf. If there exists $i \in \mathcal{G}_{+}$such that $i \mathcal{I} \operatorname{Br}(x)$ then $\operatorname{Br}(x)$ is a minimal $J E P$ for the dataset $\mathcal{G}^{\prime}=\mathcal{G}_{+} \cup\left\{j \leq l_{o b j}(x)\right\}$.

Proof. By definition of a ToMJEPs, for a node $x$, we have $\operatorname{Br}(x) \cap \mathcal{D}_{\bullet j} \neq \emptyset, \forall j \leq$ $l \leq l_{o b j}(x)$. Thanks to Prop. 1, it follows that $\operatorname{Br}(x)$ is a JEP for $\mathcal{G}_{+} \cup\{j \leq$ $\left.l_{o b j}(x)\right\}$.

If $x$ is not a dead-end leaf, by definition of a ToMJEPs, we have $\forall z \leq$ $x, \operatorname{crit}\left(l_{\text {attr }}(z), x\right) \neq \emptyset$, thus $\forall a \in B r(x), \exists j \in \cup\left\{j \leq l_{\text {obj }}(x)\right\}$ such that $\operatorname{Br}(x) \cap$ $\mathcal{D}_{\bullet} j=\{a\}$. Prop. 2 ensures that $\operatorname{Br}(x)$ is a minimal JEP for the dataset $\mathcal{G}_{+} \cup\left\{j \leq l_{o b j}(x)\right\}$.

Lemma 3. Let $(T, r)$ be a ToMJEPs. Let $p$ be pattern. If $p$ is a minimal JEP for the dataset $\mathcal{G}_{+} \cup \mathcal{G}_{-}$then there exists a unique candidate leaf $x$ such that $\operatorname{Br}(x)=p$.

Proof. The proof reasons inductively on $\mathcal{G}_{-}$. For a sake of simplicity, we denote here the set of the negative objects as $\{1, \ldots, k\}$ with $k=\left|\mathcal{G}_{-}\right|$and $\forall 1 \leq j \leq$ $k-1, j \prec j+1$.

Definition 1 implies that the children of the root $r$ deal with 1 (the first negative object), we have $\mathcal{D}_{\bullet 1}=\left\{l_{\text {attr }}(x): \quad x\right.$ is a child of $\left.r\right\}$. Moreover, as by definition of a ToMJEPs, $\operatorname{crit}\left(l_{\text {attr }}(x), x\right) \neq \emptyset$, no child of $r$ is a dead-end leaf. Thus, associated to any pattern $p$ which is a minimal JEP for the dataset $\mathcal{G}_{+} \cup\{1\}$, there is a unique node $x$, different from a dead-end leaf such that $\operatorname{Br}(x)=p$. 
Let us now suppose that, considering any minimal JEP $p$ for $\mathcal{G}_{+} \cup\{1, \ldots, l\}$ with $l<k$, there exists a unique node $x$, different from a dead-end leaf, such that $\operatorname{Br}(x)=p$. When we consider a pattern $q$, minimal JEP for the dataset $\mathcal{G}_{+} \cup\{1, \ldots, l, l+1\}$, two cases arise:

- If $q$ is a minimal JEP for $\mathcal{G}_{+} \cup\{1, \ldots, l\}$, then, thanks to the induction hypothesis, there exists a unique node $x_{q}$ such that $\operatorname{Br}\left(x_{q}\right)=q$.

- Otherwise, thanks to the consequence of Prop. 2, there exists one attribute $a$ such that $\mathcal{D}_{\bullet l+1} \cap q=\{a\}$ and $\mathcal{D}_{\bullet} \cap \cap a \neq\{a\}, \forall j \preceq l$. Prop 2 ensures that $q \backslash\{a\}$ is minimal JEP for $\mathcal{G}_{+} \cup\{1, \ldots, l\}$. Thanks to the induction hypothesis, there exists a unique node $x$, different from a dead-end leaf, such that $\operatorname{Br}(x)=q \backslash\{a\}$. By definition of a ToMJEPs, there exists a unique child of $x$, such that $\operatorname{Br}(q)=x$. As $q$ is a minimal JEP, $x$ is not a dead-end leaf.

Prop. 3 is a consequence of Lemmas 2 and 3:

Proposition 3 (One-To-One correspondence). Let (T,r) be a ToMJEPs. There is a one-to-one correspondence between the set of the candidate leaves $x$ such that $\operatorname{Br}(x)$ is a supported pattern and the set of the minimal JEPs.

Prop. 3 ensures that we can generate the minimal JEPs by simply performing a depth first traversal of a ToMJEPs and output the candidate leaves such that $B r(x)$ is a supported pattern. Note that it is not necessary to compute and store the entire ToMJEPs. A depth first traversal only requires to store the path from the root to the node currently visited.

The sketch of implemention provided in Section 4.1 gives information about the calculation of the extent, the calculation of the essential JEPs and the top- $k$ minimal JEPs that are inferred from a ToMJEPs.

\section{Experimental evaluation}

This section provides and comments results from a study conducted on 13 benchmark datasets. We investigate the computation of the JEPs according to running time, setting a minimum frequency threshold. It also indicates the reliability of correlation between a JEP and a class. In the following, a JEP denominates a supported pattern with respect to any class.

\subsection{Material and methods}

The datasets. The study is conducted on 13 usual datasets described in Table 3 . All the datasets are available from the UCI Machine Learning repository [10]. We selected these datasets because they have been used, at least once, in an experimental assessment of JEPs $[3,4,16]$. Non binary attributes were converted into a binary valued format by applying a sanctioned method $[6,11]$ which is available at Frans Coenen's website ${ }^{1}$.

\footnotetext{
${ }^{1}$ http://cgi.csc.liv.ac.uk/ frans/KDD/Software/LUCS-KDD-DN/exmpleDNnotes. html
} 
Table 3. The datasets and their characteristics

\begin{tabular}{|c|c|c|c||c|c|c|c|}
\hline Datasets & Objects & Attributes & Classes & Datasets & Objects & Attributes & Classes \\
\hline breast & 699 & 20 & 2 & mushroom & 8124 & 90 & 2 \\
congres & 435 & 34 & 2 & pima & 768 & 38 & 2 \\
ecoli & 336 & 34 & 8 & tic-tac-toe & 958 & 29 & 2 \\
glass & 214 & 48 & 7 & waveform & 5000 & 101 & 3 \\
heart & 303 & 52 & 5 & wine & 178 & 68 & 3 \\
hepatitis & 155 & 56 & 2 & zoo & 101 & 42 & 7 \\
iris & 150 & 19 & 3 & \multicolumn{2}{l}{} \\
\cline { 5 - 8 }
\end{tabular}

Implementation. Our algorithm partially explores a ToMJEPs in a depth first manner, it outputs every candidate leaf whose associated pattern is a supported one. We implemented two solutions to ensure to only output supported patterns. The first one, called post-filtering solution, generates all the candidate leaves and then checks whether their extent is empty or not. The second one, named maintaining_extent solution, integrates the computation of the extents with the calculation of the child of an internal node of a ToMPJEPs. It enables to backtrack as soon as the extent is empty.

Moreover, when a minimum frequency threshold is provided, the maintaining_extent solution is straightforwardly adapted to improve the computing of the essential JEPs. Indeed, the frequency of candidate essential JEPs [4] is directly derived from the cardinality of the extent. For the same reason, this solution also enables to compute the top- $k$ minimal JEPs [16] when a value for $k$ is provided. Moreover, the pruning strategy becomes more and more efficient during the mining step because the minimal frequency threshold to belong to the top- $k$ minimal JEP only increases during the mining.

Protocol. In order to compute all the minimal JEPs whatever the positive class is, we successively consider each class (of the dataset) as the positive class while the union of the others classes constitutes the negative class. Computations were performed on a server using Ubuntu 12.04 with 2 processors Intel Xeon $2.80 \mathrm{GHz}$ and 512 gigabytes of RAM.

\subsection{Results and discussions}

Computation of the minimal JEPs. We computed all the minimal JEPs on the 13 selected datasets, by using the post-filtering and maintaining_extent solutions. Moreover, essential JEPs are computed with two minimum frequency thresholds (1\% and 5\%), and the top- $k$ JEPs with $k=10$ and $k=20$. Table 4 gives the cardinalities of the sets of the minimal JEPs and the running times. For computing all the minimal JEPs, the maintaining_extent solution always operates faster than the post-filtering solution, by a factor varying from 1.6 to 3 . By observing the results for the essential JEPs and top-k minimal JEPs, one notes that the running time decreases significantly when a minimal threshold is set for the cardinality of the extent. The use of a frequency constraint related to the 
Table 4. Computation of minimal JEP including negation of attributes

\begin{tabular}{|c|c|c|c|c|c|c|c|}
\hline \multirow{2}{*}{} & \multicolumn{3}{|c|}{ All minimal JEPs } & \multicolumn{2}{c|}{ Essential JEPs } & \multicolumn{2}{c|}{ Top-K minimal JEPs } \\
\cline { 2 - 8 } & & post-filtering & maintaining_extent & $1 \%$ & $5 \%$ & 10 & 20 \\
\hline Datasets & Min.JEPs & Time & Time & Time & Time & Time & Time \\
\hline iris & 40 & $70.564 \mathrm{~ms}$ & $24.348 \mathrm{~ms}$ & $14.316 \mathrm{~ms}$ & $9.783 \mathrm{~ms}$ & $13.043 \mathrm{~ms}$ & $17.303 \mathrm{~ms}$ \\
breast & 38 & $924.998 \mathrm{~ms}$ & $347.572 \mathrm{~ms}$ & $190.432 \mathrm{~ms}$ & $79.198 \mathrm{~ms}$ & $95.212 \mathrm{~ms}$ & $119.213 \mathrm{~ms}$ \\
ecoli & 200 & $842.345 \mathrm{~ms}$ & $353.734 \mathrm{~ms}$ & $173.658 \mathrm{~ms}$ & $98.982 \mathrm{~ms}$ & $134.314 \mathrm{~ms}$ & $136.712 \mathrm{~ms}$ \\
zoo & 3323 & $1339.008 \mathrm{~ms}$ & $579.208 \mathrm{~ms}$ & $232.023 \mathrm{~ms}$ & $101.032 \mathrm{~ms}$ & $67.178 \mathrm{~ms}$ & $79.032 \mathrm{~ms}$ \\
pima & 1443 & $7.323 \mathrm{~s}$ & $3.093 \mathrm{~s}$ & $895.053 \mathrm{~ms}$ & $532.123 \mathrm{~ms}$ & $1.009 \mathrm{~s}$ & $1.694 \mathrm{~s}$ \\
glass & 59747 & $27.172 \mathrm{~s}$ & $12.418 \mathrm{~s}$ & $6.927 \mathrm{~s}$ & $3.241 \mathrm{~s}$ & $1.439 \mathrm{~s}$ & $2.081 \mathrm{~s}$ \\
congres & 55449 & $89.396 \mathrm{~s}$ & $38.077 \mathrm{~s}$ & $19.145 \mathrm{~s}$ & $8.380 \mathrm{~s}$ & $3.107 \mathrm{~s}$ & $4.929 \mathrm{~s}$ \\
hepatitis & 410404 & $123.520 \mathrm{~s}$ & $53.706 \mathrm{~s}$ & $25.576 \mathrm{~s}$ & $14.419 \mathrm{~s}$ & $2.978 \mathrm{~s}$ & $3.097 \mathrm{~s}$ \\
heart & 122865 & $3.351 \mathrm{mn}$ & $1.194 \mathrm{mn}$ & $29.560 \mathrm{~s}$ & $15.201 \mathrm{~s}$ & $9.432 \mathrm{~s}$ & $8.921 \mathrm{~s}$ \\
tic-tac-toe & 109949 & $5.664 \mathrm{mn}$ & $2.797 \mathrm{mn}$ & $55.860 \mathrm{~s}$ & $13.182 \mathrm{~s}$ & $4.541 \mathrm{~s}$ & $6.325 \mathrm{~s}$ \\
wine & 1353996 & $200.321 \mathrm{mn}$ & $99.366 \mathrm{mn}$ & $58.053 \mathrm{mn}$ & $36.324 \mathrm{mn}$ & $8.342 \mathrm{mn}$ & $11.821 \mathrm{mn}$ \\
mushroom & 17345228 & $673.563 \mathrm{mn}$ & $423.116 \mathrm{mn}$ & $192.743 \mathrm{mn}$ & $101.765 \mathrm{mn}$ & $27.545 \mathrm{mn}$ & $50.325 \mathrm{mn}$ \\
waveform & 23895434 & $1845.431 \mathrm{mn}$ & $954.190 \mathrm{mn}$ & $421.813 \mathrm{mn}$ & $238.425 \mathrm{mn}$ & $47.342 \mathrm{mn}$ & $59.175 \mathrm{mn}$ \\
\hline
\end{tabular}

cardinality of the extent is efficient, obviously there is the risk to miss interesting patterns.

Minimal JEPs as rules to express correlations. A JEP expresses a correlation between the occurrence of a pattern and one class of objects. This part provides experimental results to assess the interest of such rules: do these rules cover a large part of the objects? Are they confident enough? We have also performed experiments to evaluate the usefulness of the explicit description of the absent attributes by adding their negations.

The study has been conducted by using a leave-one-out framework: every object has been successively discarded from the dataset. For every object $g$, the minimal JEPs have been extracted by considering $\mathcal{G} \backslash\{g\}$ as the dataset and the resulting rules have been applied on $g$.

Table 5 provides results obtained by applying minimal JEPs, essential JEPs, or top-k minimal JEPs as association rules. No Negated attributes designates the descriptions which do not explicitly take into account the absence of attributes whereas With Negated attributes points the descriptions that explicitly consider the absence of attributes. The column Cov denotes the coverage of the set of association rules (the part of the objects for which at least one association rule has applied). The column Con refers to the average confidence (i.e., the ratio between the number of correct applications of the rules over the whole number of applications of the rules). For example, if we consider the dataset named breast, whith the No Negated attributes description, $47.78 \%$ of the objects contain at least one minimal JEP, this coverage raises to $49.33 \%$ of the objects when the descriptions With Negated attributes are accounted. With the same dataset, by using the No Negated attributes description, $98.19 \%$ of the rules resulting from a minimal JEP apply on an object of the proper class ; this average confidence slightly decreases to $96.13 \%$ when the No Negated attributes description is used. 
First of all, the JEPs often apply on a large portion of the objects: for 7 datasets among the 13 datasets, more than $80 \%$ of the objects contain at least one JEP. Note that this coverage increases when the description turns from No Negated attributes to With Negated attributes, up to $8 \%$ for the hepatitis dataset.

The average confidences indicate that minimal JEPs often point a reliable association between a pattern and a class, even when no frequency constraint is set. By paying the price of a lower coverage, setting a minimum frequency threshold - as it is done for the essential JEPs or, indirectly, for the top- $k$ minimal JEPs - causes an increase of the average confidence, depending on the dataset. The average confidence levels reached by the two descriptions, No Negated attributes and With Negated attributes, are very comparable.

As a conclusion, both description families, With Negated attributes and No Negated attributes, lead to minimal JEPs reaching a similar level of confidence. However, the minimal JEPs extracted with the With Negated attributes descriptions cover a wider range of objects than the minimal JEPs extracted with the No Negated attributes descriptions, but with a longer running time.

\section{Related work}

Since the key paper of Dong and Li [2], subsequent research has focused on mining emerging patterns and contrast sets. However, there are very few attempts to tackle the discovery of minimal JEPs. Fan and Ramamohanarao have proposed an algorithm extracting the minimal JEPs whose frequency of occurrence is greater than a given threshold, such JEPs are called essential JEPs [4]. Terlecki and Walczak have designed a computational method based on a CP-Tree to get the $k$ most supported minimal JEPs, named top- $k$ minimal JEPs [16]. These methods require either a frequency threshold or a given number of expected patterns. On the contrary, our method is free from these parameters and computes the whole set of minimal JEPs. Terlecki and Walczak [15] have experimentally shown that taking into account the absence of attributes may provide interesting pieces of knowledge to build more accurate classifiers. We have dealt with this issue since our method extracts minimal JEPs including the negation of the attributes which are absent.

In addition, JEPs can be associated to version space [13]. A version space gathers the descriptions that match all objects of one class and no object of the other class. Therefore a version space corresponds to the JEPs that match all objects of one class. JEPs are also related to the concept of disjunctive version space since a JEP corresponds to all descriptions of objects that match at least one object of one class and no object for the other classes. In Formal Concept Analysis, a JEP is also named "hypothesis" [5] (a hypothesis brings together the descriptions of objects that match at least one object in one class and no object in others). 
Table 5. Evaluation of minimal JEPs as rules to express correlations

\begin{tabular}{|c|c|c|c|c|c|c|c|c|c|c|c|c|c|c|c|c|}
\hline ¿ & & 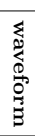 & $\begin{array}{l}\text { t. } \\
i \\
0 \\
0 \\
i \\
0 \\
0\end{array}$ & . & $\begin{array}{l}\text { है } \\
\text { है } \\
0 \\
0\end{array}$ & $\stackrel{5:}{*}$ & 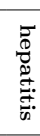 & בֶّ & 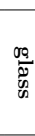 & $\stackrel{0}{\stackrel{\delta}{ٍ}}$ & 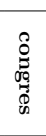 & \begin{tabular}{l}
$\sigma$ \\
$\sigma$ \\
$\tilde{D}$ \\
\multirow{2}{*}{}
\end{tabular} & 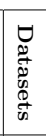 & & & \\
\hline$\stackrel{\infty}{\Xi}$ & $\begin{array}{l}\vec{N} \\
0 \\
0\end{array}$ & 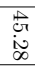 & $\stackrel{\infty}{\stackrel{\infty}{\infty}}$ & $\begin{array}{l}\vec{H} \\
\dot{0}\end{array}$ & de & $\begin{array}{l}\infty \\
\infty \\
0 \\
\vdots \\
-1\end{array}$ & $\begin{array}{l}\text { D } \\
\stackrel{N}{0} \\
\end{array}$ & 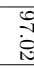 & $\underset{\infty}{\stackrel{\infty}{\infty}}$ & 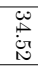 & $\begin{array}{l}8 \\
\vdots \\
\infty\end{array}$ & $\begin{array}{l}\vec{A} \\
-1 \\
\infty\end{array}$ & 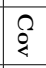 & & \multirow{2}{*}{ 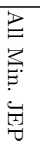 } & \\
\hline 总 & 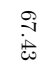 & 予 & \begin{tabular}{l}
$\infty$ \\
$\infty$ \\
$\dot{d n}$ \\
\cline { 1 - 1 }
\end{tabular} & $\underset{\mathrm{i}}{\stackrel{c}{\mathrm{~N}}}$ & i & $\begin{array}{l}\stackrel{0}{\circ} \\
\stackrel{\circ}{\infty}\end{array}$ & 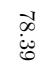 & 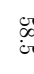 & 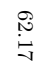 & 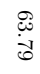 & 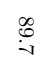 & $\begin{array}{l}0 \\
0 \\
0 \\
0\end{array}$ & @ & & & \\
\hline 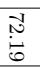 & & 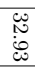 & 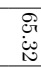 & 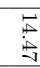 & \begin{tabular}{ll}
\multicolumn{1}{c}{} \\
\\
\end{tabular} & $\begin{array}{l}7 \\
6 \\
0 \\
0\end{array}$ & 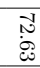 & لئن & 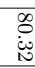 & 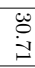 & $\begin{array}{l}0 \\
\stackrel{0}{0} \\
\stackrel{0}{0}\end{array}$ & 虫 & 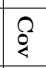 & & & \multirow{8}{*}{ 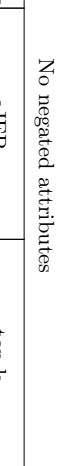 } \\
\hline 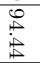 & $\vec{U}$ & $\frac{\vec{T}}{0}$ & $\begin{array}{l}\infty \\
\infty \\
\dot{0}\end{array}$ & 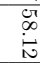 & ( & 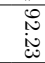 & ${ }_{0}^{\infty}$ & 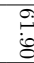 & $\stackrel{i}{\infty}_{\infty}^{\prime \prime}$ & $\begin{array}{l}\stackrel{2}{d} \\
\dot{d}\end{array}$ & $\begin{array}{l}0 \\
\stackrel{0}{0} \\
\infty\end{array}$ & $\begin{array}{l}0 \\
\dot{w} \\
\dot{w}\end{array}$ & $\S$ & d9 & & \\
\hline $\begin{array}{l}8 \\
\stackrel{8}{0}\end{array}$ & & $\begin{array}{l}\mathbb{1} \\
0 \\
:\end{array}$ & $\stackrel{2}{\mathscr{\omega}}$ & 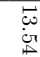 & d & $\begin{array}{l}\overrightarrow{i v} \\
\text { du }\end{array}$ & 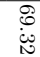 & 象 & $\begin{array}{l}\text { ज } \\
\dot{8}\end{array}$ & 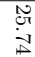 & $\begin{array}{l}\int_{0}^{\infty} \\
\dot{U} \\
\end{array}$ & $\underset{\infty}{\stackrel{t}{\infty}}$ & 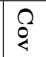 & & & \\
\hline 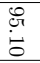 & & $\begin{array}{l}\vec{v} \\
\overrightarrow{0}\end{array}$ & 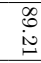 & $\stackrel{\circ}{\circ}$ & $\begin{array}{l}\infty \\
\alpha \\
d \\
d \\
d\end{array}$ & $\begin{array}{l}\circ \\
\dot{0}\end{array}$ & $\stackrel{\infty}{\stackrel{\infty}{b}}$ & 商 & 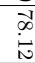 & $\overrightarrow{\vec{d}}$ & $\begin{array}{l}\stackrel{\stackrel{0}{0}}{\stackrel{\omega}{\dot{\omega}}} \\
\end{array}$ & $\begin{array}{l}8 \\
8 \\
6\end{array}$ & $\stackrel{\varrho}{g}$ & 39 & & \\
\hline 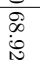 & & 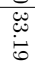 & $\begin{array}{l}\text { gे } \\
\stackrel{d}{\omega}\end{array}$ & $\begin{array}{l}\vec{\omega} \\
\dot{\omega}\end{array}$ & $\begin{array}{l}\vec{c} \\
\stackrel{\alpha}{\alpha} \\
\dot{\alpha}\end{array}$ & $\vec{g}$ & $\begin{array}{l}08 \\
0 \\
0\end{array}$ & did & $\begin{array}{l}\text { ते } \\
\text { dै }\end{array}$ & 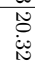 & $\overrightarrow{⿱ 乛 龰}$ & 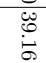 & $\oint_{8}$ & \multirow[b]{2}{*}{$\vec{\sigma}$} & & \\
\hline 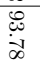 & $\begin{array}{l}1 \\
\infty \\
\infty \\
\infty\end{array}$ & $\begin{array}{l}\text { نे } \\
\dot{b}\end{array}$ & $\begin{array}{l}\text { o } \\
\stackrel{\text { in }}{*}\end{array}$ & 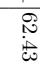 & $\underset{\alpha}{\alpha}$ & $\begin{array}{l}\mathscr{0} \\
\dot{6}\end{array}$ & 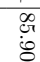 & D. & $\vec{d}$ & $\begin{array}{l}\vec{y} \\
\vec{c}\end{array}$ & $\begin{array}{l}0 \\
\stackrel{0}{0} \\
-1\end{array}$ & $\begin{array}{l}0 \\
0 \\
\infty \\
i \pi\end{array}$ & $\S$ & & 5 & \\
\hline 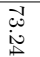 & 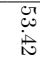 & 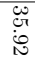 & 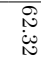 & $\underset{\vec{d}}{\vec{c}}$ & 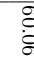 & $\underset{\infty}{\vec{d}}$ & N & 恿 & $\begin{array}{l}\overrightarrow{i v} \\
i \\
\text { i }\end{array}$ & 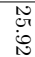 & $\begin{array}{l}\infty^{\prime} \\
\dot{c} \\
\dot{c}\end{array}$ & $\begin{array}{l}\vec{c} \\
\dot{u} \\
\dot{0}\end{array}$ & $\S$ & \multirow{2}{*}{$\stackrel{0}{0}$} & 䕗 & \\
\hline $\begin{array}{l}\infty \\
\infty \\
-1 \\
0 \\
j\end{array}$ & 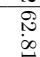 & Nָ & Do & $\begin{array}{l}\text { I } \\
\infty \\
0\end{array}$ & de & $\stackrel{\circ}{\stackrel{0}{\circ}}$ & $\begin{array}{l}\infty \\
0 \\
0 \\
0\end{array}$ & : & $\begin{array}{l}\infty \\
\infty \\
\dot{0}\end{array}$ & $\begin{array}{l}\mathscr{D} \\
0 \\
0\end{array}$ & $\stackrel{\leftrightarrow}{0}$ & $\begin{array}{l}0 \\
0 \\
0\end{array}$ & $\S$ & & & \\
\hline 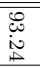 & 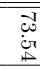 & $\begin{array}{l}\vec{\infty} \\
\infty \\
\dot{6}\end{array}$ & $\begin{array}{l}\infty \\
\stackrel{\infty}{N} \\
\stackrel{N}{N}\end{array}$ & $\begin{array}{l}\vec{v} \\
\dot{j} \\
\dot{i} \\
\end{array}$ & \begin{tabular}{l}
\multicolumn{1}{l}{} \\
0 \\
0
\end{tabular} & 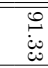 & 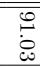 & 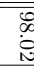 & 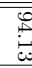 & 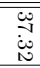 & $\begin{array}{l}\ddot{8} \\
\dot{8}\end{array}$ & 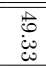 & \& & \multirow{2}{*}{\multicolumn{2}{|c|}{ 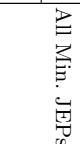 }} & \multirow{12}{*}{ 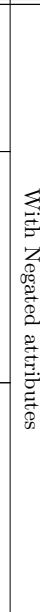 } \\
\hline 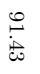 & $\begin{array}{l}\stackrel{\mathscr{H}}{\vec{j}} \\
\dot{0}\end{array}$ & $\begin{array}{l}\vec{\partial} \\
\dot{\infty}\end{array}$ & 离 & 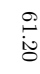 & d & 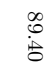 & 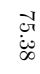 & i & $\vec{G}$ & ت己 & $\begin{array}{l}\infty \\
\stackrel{\infty}{\infty} \\
\stackrel{0}{0}\end{array}$ & $\stackrel{\mathscr{L}}{.}$ & $\stackrel{\varrho}{8}$ & & & \\
\hline 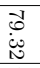 & $\begin{array}{l}\stackrel{\circ}{0} \\
\stackrel{0}{0}\end{array}$ & $\begin{array}{l}\text { 苂 } \\
\dot{g}\end{array}$ & $\begin{array}{l}\text { Dे } \\
0 \\
0\end{array}$ & $\begin{array}{l}\vec{\sigma} \\
\dot{d}\end{array}$ & d & 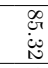 & 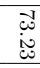 & 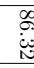 & 冓 & 离 & $\begin{array}{l}0 \\
i \\
i \\
\infty \\
\infty\end{array}$ & $\begin{array}{l}\vec{N} \\
\text { 点 }\end{array}$ & $\oint_{2}$ & \multirow{3}{*}{$\overrightarrow{8}$} & & \\
\hline $\mathbb{1}$ & I্] & $\infty^{-1}$ & 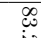 & 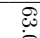 & de & 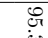 & $\overline{0}$ & $\stackrel{v}{-1}$ & 8 & $\vec{J}$ & 8 & i) & $\delta$ & & & \\
\hline $\overrightarrow{\vec{t}}$ & $\stackrel{\infty}{\circ}$ & $\stackrel{0}{0}$ & $\stackrel{i}{\sim}$ & 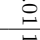 & 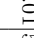 & 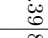 & $\vec{v}$ & 秀 & 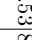 & $\dot{\infty}$ & 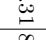 & 8 & 5 & & 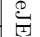 & \\
\hline 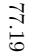 & 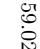 & $\begin{array}{c}c \\
\text { co } \\
\text { in }\end{array}$ & $\begin{array}{l}\text { in } \\
\stackrel{i}{t}\end{array}$ & $\stackrel{\vec{P}}{\dot{b}}$ & 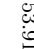 & 悥 & 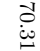 & 我 & $\overbrace{\substack{i \\
i}}^{\infty}$ & $\begin{array}{l}\tilde{\mathscr{\omega}} \\
\stackrel{\omega}{\omega}\end{array}$ & 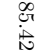 & 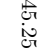 & @ & \multirow[b]{2}{*}{ dr } & & \\
\hline 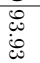 & $\begin{array}{l}\text { o. } \\
\dot{6}\end{array}$ & 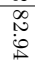 & $\underset{\substack{\infty \\
\stackrel{\infty}{\infty}}}{\infty}$ & 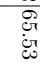 & 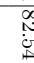 & 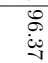 & $\underset{\substack{\infty \\
\infty \\
\infty \\
\infty}}{\infty}$ & 递 & $\stackrel{\infty}{\infty}$ & $\stackrel{\text { i }}{\mathrm{N}}$ & $\stackrel{\mathscr{B}}{\stackrel{0}{\omega}}$ & $\begin{array}{l}0 \\
0 \\
0\end{array}$ & 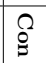 & & & \\
\hline de & $\begin{array}{l}\text { g] } \\
\dot{c} \\
\dot{\omega}\end{array}$ & 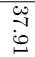 & Nu & $\underset{D}{\vec{D}}$ & $\begin{array}{l}0 \\
\dot{a} \\
i\end{array}$ & $\begin{array}{l}1 \\
0 \\
0 \\
0\end{array}$ & 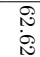 & $\stackrel{3}{\mathrm{i}}$ & $\vec{d}$ & 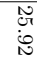 & $\begin{array}{l}\stackrel{8}{0} \\
\dot{d}\end{array}$ & $\begin{array}{l}\infty \\
\infty \\
0 \\
0\end{array}$ & $\oint_{q}$ & \multirow[b]{2}{*}{5} & & \\
\hline 苾 & i. & ढै & $\ddot{0}$ & 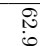 & $\stackrel{\alpha}{g}$ & $\begin{array}{l}0 \\
\text { i }\end{array}$ & $\stackrel{\infty}{0}$ & $\stackrel{\infty}{g}$ & $\vec{i}$ & गै & $\begin{array}{l}0 \\
\text { i } \\
\text { i }\end{array}$ & 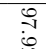 & $\S$ & & & \\
\hline$\therefore$ & $\stackrel{P}{\circ}$ & bे & जे & $\vec{u}$ & g & 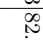 & ]$^{\prime}$ & 9 & $\infty$ & N & d' & 8 & $\Omega$ & & $\frac{T}{n}$ & \\
\hline$\dot{u}$ & $\dot{8}$ & $\dot{\vec{v}}_{\vec{v}}$ & ن̀ & نં & ఏ & $\dot{\sigma}$ & 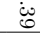 & $\dot{\Xi}$ & 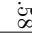 & $\dot{\omega}$ & $\dot{5}$ & iv & 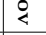 & 10 & & \\
\hline 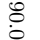 & $\stackrel{\mathrm{I}}{0}$ & $\stackrel{T}{T}$ & Iิ & G] & i & $\%$ & $\mathrm{y}$ & $\sqrt{2}$ & & $\stackrel{T}{P}$ & $\stackrel{\mathscr{L}}{\not}$ & \begin{tabular}{c}
$\mathscr{H}$ \\
\hdashline
\end{tabular} & $\delta$ & 0 & & \\
\hline
\end{tabular}

\section{Conclusion}

We have introduced an efficient method to extract the whole set of minimal JEPs. To the best of our knowledge, it is the first method which does not require either a frequency threshold or a given number of expected patterns. Our method is also able to straightforwardly extract the essential JEPs and the $k$ most supported minimal JEPs. Moreover it enables the integration of negated attributes that can be precious for a classification purpose. We have experimentally analyzed 
the computation of these JEPs, together with the reliability of the correlations between a JEP and a class.

The structure of tree of the minimal JEPs constitutes a framework for designing and expressing algorithms to compute the minimal JEPs from a dataset. In order to speed up the calculation, this framework will be used to seek for efficient orderings on the attributes or on the objects. Another direction is to produce patterns correlated to one class to a lesser extent and mine emerging patterns with high growth-rate values. Beyond this work, we plan to use minimal JEPs in the design of an advanced rule-based classifier.

\section{References}

1. Chen, X., Chen, J.: Emerging patterns and classification algorithms for dna sequence. JSW 6(6), 985-992 (2011)

2. Dong, G., Li, J.: Efficient mining of emerging patterns: Discovering trends and differences. In: KDD. pp. 43-52 (1999)

3. Dong, G., Li, J.: Mining border descriptions of emerging patterns from dataset pairs. Knowl. Inf. Syst. 8(2), 178-202 (2005)

4. Fan, H., Ramamohanarao, K.: An efficient single-scan algorithm for mining essential jumping emerging patterns for classification. In: PAKDD. pp. 456-462 (2002)

5. Ganter, B., Kuznetsov, S.O.: Hypotheses and version spaces. In: ICCS. pp. 83-95 (2003)

6. Kerber, R.: Chimerge: Discretization of numeric attributes. In: Swartout, W.R. (ed.) AAAI. pp. 123-128. AAAI Press / The MIT Press (1992)

7. Kobylinski, L., Walczak, K.: Spatial emerging patterns for scene classification. In: ICAISC (1). pp. 515-522 (2010)

8. Kobylinski, L., Walczak, K.: Efficient mining of jumping emerging patterns with occurrence counts for classification. T. Rough Sets 13, 73-88 (2011)

9. Li, J., Dong, G., Ramamohanarao, K.: Making use of the most expressive jumping emerging patterns for classification. In: PAKDD. pp. 220-232 (2000)

10. Lichman, M.: UCI machine learning repository (2013), http://archive.ics.uci. $\mathrm{edu} / \mathrm{ml}$

11. Liu, H., Setiono, R.: Feature selection via discretization. IEEE Trans. Knowl. Data Eng. 9(4), 642-645 (1997)

12. Lozano, S., Poezevara, G., Halm-Lemeille, M.P., Lescot-Fontaine, E., Lepailleur, A., Bissell-Siders, R., Cremilleux, B., Rault, S., Cuissart, B., Bureau, R.: Introduction of Jumping Fragments in Combination with QSARs for the Assessment of Classification in Ecotoxicology. J. Chem. Inf. Model. 50(8), 1330-1339 (2010)

13. Mitchell, T.M.: Generalization as search. Artif. Intell. 18(2), 203-226 (1982)

14. Novak, P.K., Lavrac, N., Webb, G.I.: Supervised descriptive rule discovery: A unifying survey of contrast set, emerging pattern and subgroup mining. Journal of Machine Learning Research 10, 377-403 (2009)

15. Terlecki, P., Walczak, K.: Jumping emerging patterns with negation in transaction databases classification and discovery. Inf. Sci. 177(24), 5675-5690 (2007)

16. Terlecki, P., Walczak, K.: Efficient discovery of top-k minimal jumping emerging patterns. In: RSCTC. pp. 438-447 (2008)

17. Wrobel, S.: An algorithm for multi-relational discovery of subgroups. In: PKDD. pp. 78-87 (1997) 